\section{Top authors in dermatology and beyond: A personal note}

A few weeks, a friend of mine directed me to a paper in the Blue Journal, the Journal of the American Academy of Dermatology, and congratulated me: I was ranked in the paper among the first 25 top authors in Dermatology by career-long total citations 1996-2019. ${ }^{1}$

I was incredulous and surprised, and still consider the ranking too generous, and not reflecting the actual merits of many authors not included in the list.

On the other hand, whatever the ranking was, the paper prompted me to some reflections on the role of research which I am willing to share with the readers of Dermatology Reports.

1. When confronted with uncertainties in clinical decisions, research implementation should represent the most normal way to practice medicine and to address unsolved questions. This was the valuable teaching of my mentor, Dr. Gianni Tognoni who considered clinical practice as the "natural laboratory" of any clinician. ${ }^{2}$ It is a way of thinking promoted by giants in Medicine such as Claude Bernard, Archibald Cochrane and Thomas Chalmers. $^{3-5}$ Even well documented case reports can help posing questions, laying the foundations for more structured research activities. A teaching example, is provided by the reporting of unexpected adverse events to newly introduced drugs which may generate signals to be explored by structured research methods.

2. Following from the above consideration, research activities should not be restricted to the academic world. I am not an academic dermatologist (one may well wonder why, see Kumar 2018 ), but have been always practicing research. Clinical research is a matter of good hypotheses, organization, networking, and multidisciplinary collaboration. Many examples of excellent research papers come from nonacademic dermatologists. In my opinion, one of the best clinical research setting in our discipline is the Research Unit of the Academia Española de Dermatología y Venereología which is not stricto sensu an academic milieu and is led by Dr. Ignacio Garcia Doval a clever and, as far as I know, a hospital dermatologist. ${ }^{7}$ In my opinion, there should be ways to promote research in clinical practice outside academic institutions, and to reward people dedicating time to research in such a context.

Our journal is run by the Italian Association of Hospital Dermatologists (ADOI), which is an organization mainly grouping non-academic hospital dermatologists. We should be able to promote a culture of research in our discipline with a strong foundation in clinical practice.

Looking forward to receiving your contributions!

Luigi Naldi UOC Dermatologia, Ospedale San Bortolo, Vicenza; Centro Studi GISED, Bergamo, Italy

\section{References}

1. Maymone MBC, Dellavalle RP. Top authors in dermatology: Comparisons
Correspondence: Luigi Naldi, UOC Dermatologia, Ospedale San Bortolo, Vicenza; Centro Studi GISED, Bergamo, Italy

Received for publication: 25 May 2021

Accepted for publication: 25 May 2021.

This work is licensed under a Creative Commons Attribution-NonCommercial 4.0 International License (CC BY-NC 4.0).

(Copyright: the Author(s), 2021

Licensee PAGEPress, Italy

Dermatology Reports 2021; 13:9274

doi:10.4081/dr.2021.9274

of standardized database citation indicators. J Am Acad Dermatol 2021:S01909622.

2. Tognoni G, Bonati M. Second-generation clinical pharmacology. Lancet 1986;2: 1028-9.

3 Claude Bernard, La Science Expérimentale, París, Baillière, 1878.

4. Cochrane AL Effectiveness and efficiency: Random reflections on health services. Nuffield Trust, 1972.

5. Dickersin K, Chalmers F. Thomas C Chalmers (1917-1995): a pioneer of randomised clinical trials and systematic reviews. J R Soc Med 2015;108:23741.

6. Kumar V D. Academic nepotism - all that glitters is not gold. J Adv Med Educ Prof 2018;6:186-7.

7. Molina-Leyva A, Descalzo MA, García-Doval I. A Map of Clinical Dermatology Research Centers in Spain: Results of the MaIND Study. Actas Dermosifiliogr 2017;108:830-5. 